\title{
Inner tracking performance in ATLAS: vertex and mass resolution
}

\author{
Ina Chalupkova ${ }^{* \dagger}$ on behalf of the ATLAS Collaboration \\ Charles University in Prague, Institute of Particle and Nuclear Physics \\ E-mail: Ina.Chalupkova@cern.ch
}

The ATLAS Inner Detector track reconstruction showed an excellent performance during Run 1 of the LHC from 2009 to 2013, while the increasing number of instantaneous proton-proton collisions created a more and more challenging environment during this period. In particular, the reconstruction of primary and secondary vertices has proven to cope very well in this environment and has been an integral part of many physics analyses performed with ATLAS data. We present an overview on the general track reconstruction performance with a dedicated focus on vertex and short-lived particle reconstruction. The latter has been used in many physics analyses, and was also an important constraint for detector alignment and to establish momentum scale and material budget systematics of the inner tracking system.

14th International Conference on B-Physics at Hadron Machines,

April 8-12, 2013

Bologna, Italy

\footnotetext{
* Speaker.

$\dagger$ The author acknowledges support of grant MSM0021620859 of Ministry of Education, Youth and Sports of the Czech Republic.
} 

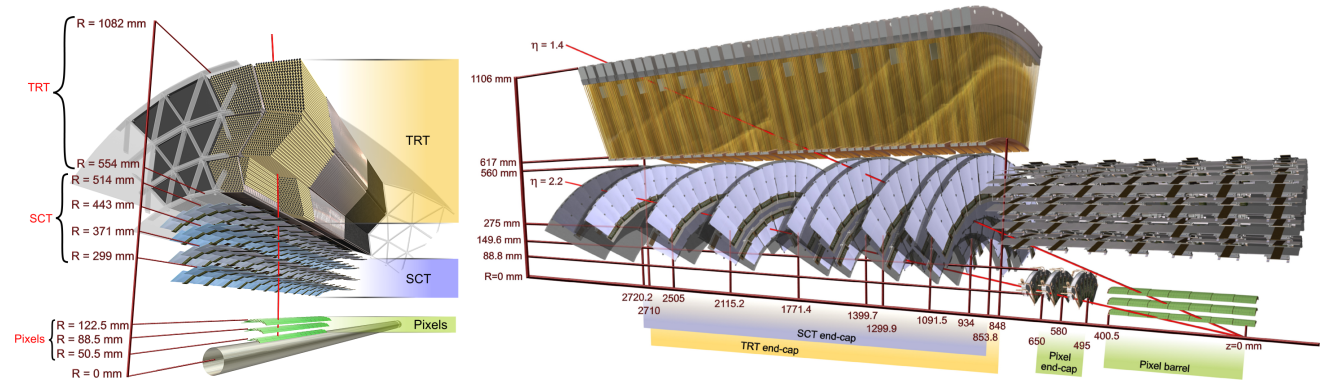

Figure 1: Schematic diagrams showing the structure of barrel (left) and end-caps of ATLAS Inner Detector.

\section{The ATLAS Inner Detector}

The ATLAS detector [1] at the Large Hadron Collider [2] consists of four major components: the Inner Detector (ID), electromagnetic and hadronic calorimeters, muon spectrometer and magnet system. The ID tracker provides precision measurements of charged particle trajectories, vertex reconstruction, excellent b-tagging in jets and particle identification. It consists of three subdetectors: a silicon pixel detector closest to the interaction point; the silicon strip SemiConductor Tracker (SCT); and the Transition Radiation Tracker (TRT), a drift chamber with gas-filled straw tubes. All sub-systems are divided in three sections: a barrel composed of several cylindrical layers and two end-caps consisting of disks (wheels) of modules. The whole ID is contained in a $2 \mathrm{~T}$ axial magnetic field provided by a superconductive central solenoid.

An accurate description of geometry and position of each detector element is essential for precise measurement of track parameters. The ID alignment is performed in three steps, each of them with more detailed detector structure: first level takes sub-detector barrels and end-caps (7 elements), second considers separately barrel layers and end-cap disks (200 objects) and the last level aligns each silicon module or TRT straw $\left(3.5 \times 10^{5}\right.$ objects and $7.3 \times 10^{5}$ degrees of freedom). It is using a track-based method which is minimising a $\chi^{2}$ of hit to track residuals. The alignment is monitored on run-by-run basis and limited movements of about $5 \mu \mathrm{m}$ on the first level can be usually correlated to changes in detector temperature or other conditions.

\section{Track and Vertex Reconstruction}

Track reconstruction uses a series of pattern recognition algorithms. Tracks produced directly from the proton collisions are reconstructed with an inside-out algorithm which begins with 3point seeds built from hits in the silicon detectors. These seeds are then extended to all layers in the silicon detector to search for additional hits using a combinatorial Kalman filter. Finally silicon tracks are matched to TRT track segments. Reconstructed tracks are required to have a transverse momentum $p_{\mathrm{T}}>400 \mathrm{MeV}$. Recovery of secondary tracks from conversions, material interactions and long-lived particle decays is achieved through a back-tracking algorithm taking clusters from TRT track segments and extending tracks inwards.

Vertex reconstruction algorithm takes a seed, position of which is determined by the global maximum of the $z_{0}$ (position at beam-line) of tracks weighted according to their $p_{\mathrm{T}}$, and iteratively 

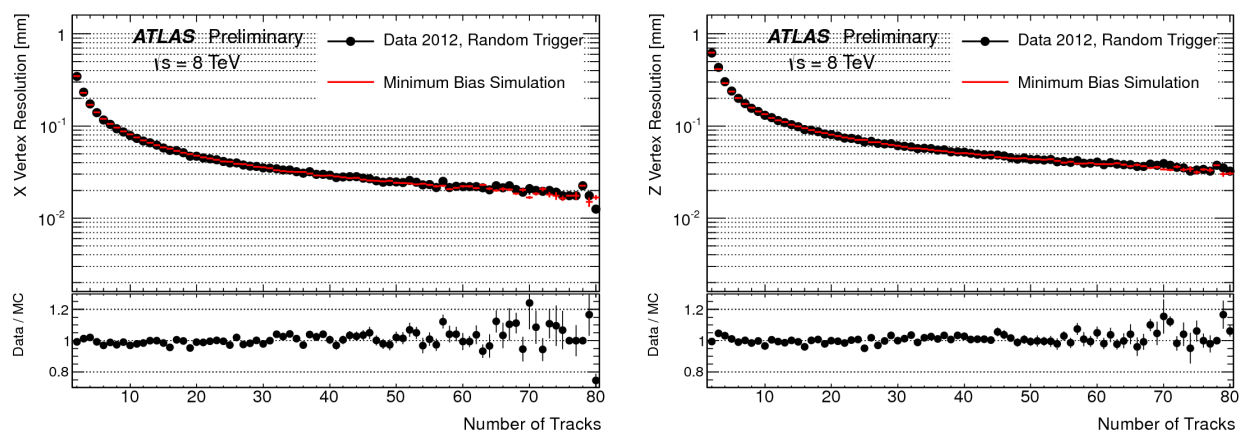

Figure 2: Vertex position resolution in $8 \mathrm{TeV}$ data (collected in with minimum bias trigger with low pile-up) and $\mathrm{MC}$ for the transverse (left) and longitudinal coordinate as a function of number of tracks in vertex [3].

fits tracks consistent with this interaction region using a $\chi^{2}$-based algorithm and the beam spot as a constraint. In the next iteration, a new vertex is seeded if a track is displaced by more than $7 \sigma$ from existing vertices and tracks can be reassigned to better-matching vertices. The primary vertex is selected as the vertex with the highest assigned $\sum p_{\mathrm{T}}^{2}$ of tracks.

The performance of vertex reconstruction in data is measured with a split vertex technique: by randomly splitting the vertices in two, each with approximately half the original tracks and similar $p_{\mathrm{T}}$ distribution, it is possible to use their separation to get an estimate of their combined intrinsic resolution. Figure 2 shows the primary vertex resolution in transverse and longitudinal direction in data, compared to Monte Carlo simulation. Resolutions achieved for vertices with 70 tracks are $\sim 23 \mu \mathrm{m}$ in transverse direction and $\sim 40 \mu \mathrm{m}$ along the beam axis [3].

\section{High Pile-up Performance}

The ID performance is particularly sensitive to particle multiplicity due to the combinatorial nature of track finding. The high detector occupancy can degrade track parameter resolution and track efficiency as well as increase fake track rate by random combinations of hits. Higher number of fake tracks in high pile-up also increases the probability to reconstruct a fake vertex.

To mitigate these effects, a set of cuts has been developed in early 2012 (so called robust requirements). It requires 9 hits and zero holes (no hit in detector where the track was reconstructed), without the need to increase the default cut on track $p_{\mathrm{T}}>400 \mathrm{MeV}$. Figure 3 and Figure 4 show that the new algorithm reduces the amount of non-primary tracks (secondary and fake tracks) in primary vertices by a factor of 2-5 while decreasing vertex reconstruction efficiency by no more than 5\%. It also improves stability of track and vertex efficiency against pile-up up to $\mu=40$ [4].

\section{Alignment-related Systematic Effects on the ID tracking}

Analysis of data from 2011 and Monte Carlo shown that there exist track parameter biases caused by systematic deformations which cannot be removed using a track-based alignment alone. Therefore systematics on tracking and measured momentum are studied measuring invariant mass of some known particles in their decays to opposite-sign tracks, e.g. $J / \psi \rightarrow \mu^{+} \mu^{-}$or $Z \rightarrow \mu^{+} \mu^{-}$ 

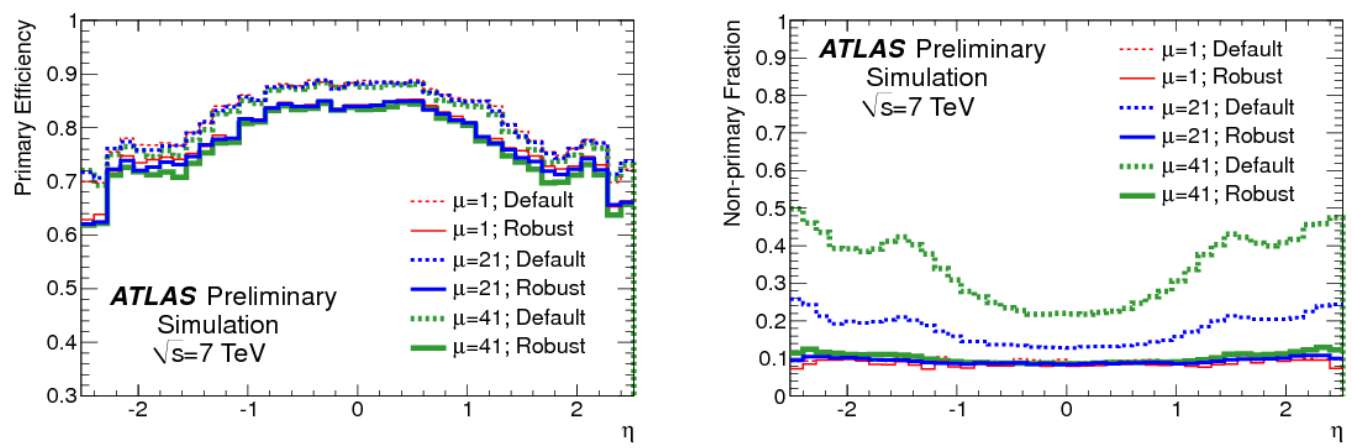

Figure 3: Primary track reconstruction efficiency (left) and the non-primary fraction (secondary and fake tracks, right) as a function of pseudorapidity in minimum bias MC samples containing one or on average 21 or 41 interactions [4].
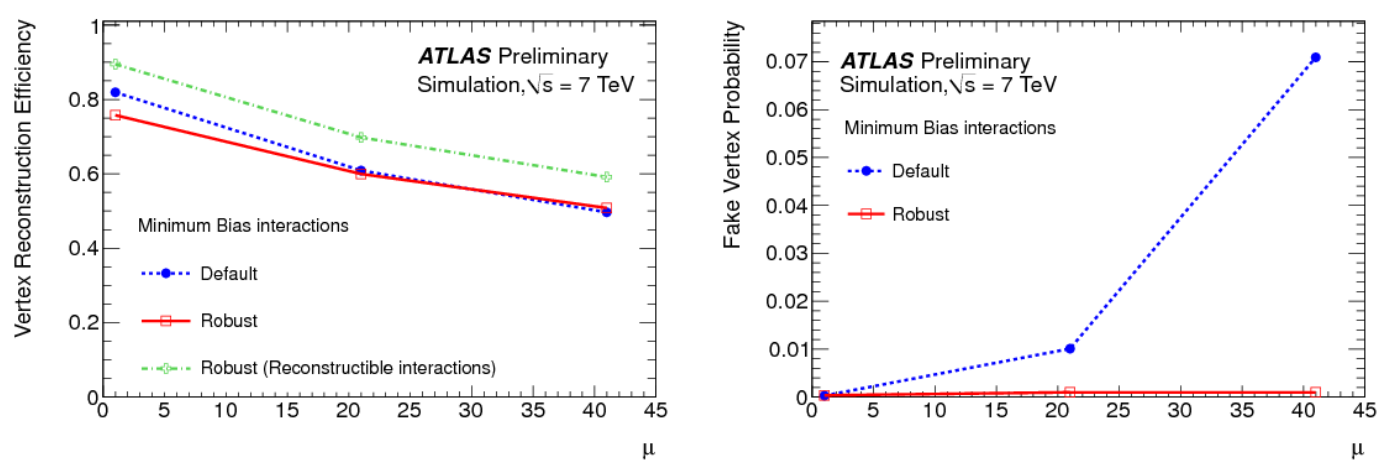

Figure 4: Vertex reconstruction efficiency (left) and fake probability (right) as a function of the average number of interactions in minimum bias MC [4].

[5]. Figure 5 shows an example of a significant improvement of $Z$ mass resolution after corrections of measured systematic biases were applied.

Second approach was to compute the ratio $E / p$ of electron energy measured by ATLAS calorimeters and momentum measured in the ID. The momentum bias was extracted from data using electrons and positrons from $Z$ and $W$ decays using $E / p$ method with parametrization of momentum $q / p=q / p\left(1+q p_{\mathrm{T}} \delta_{\text {sagitta }}\right)$. When integrated over a whole detector region, the observed residual $\delta$ bias did not exceed $0.03 \mathrm{TeV}^{-1}$ corresponding to an upper limit on the momentum bias of $0.12 \%$ at $40 \mathrm{GeV}$. However, for few isolated spots in very forward region local biases may be as large as $|\delta|=0.5 \mathrm{TeV}^{-1}$ (less than $2 \%$ bias at $40 \mathrm{GeV}$ ), as can be seen in Figure 6.

\section{Conclusions}

The excellent performance of ATLAS Inner Detector tracking in Run 1 of the LHC was based on a detailed understanding of track and vertex reconstruction and detector alignment. The impact of increasing pile-up has been studied and various techniques were implemented to maintain the 

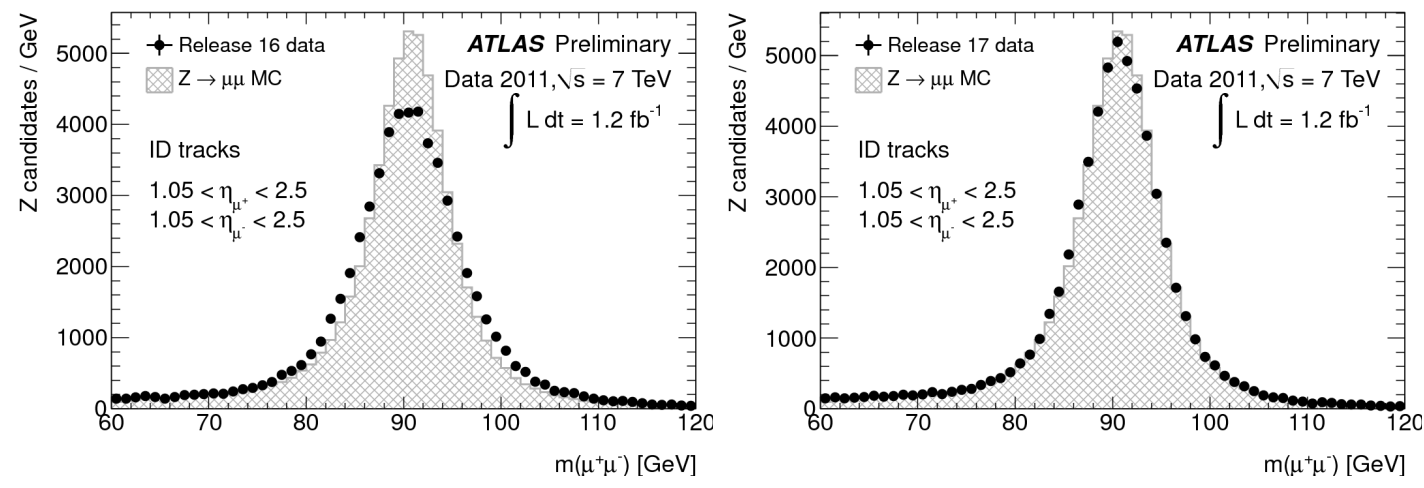

Figure 5: Reconstructed $Z$ mass distributions before (left) and after alignment corrections and perfectly aligned MC (grey) when both muons are in the end-cap A [5].
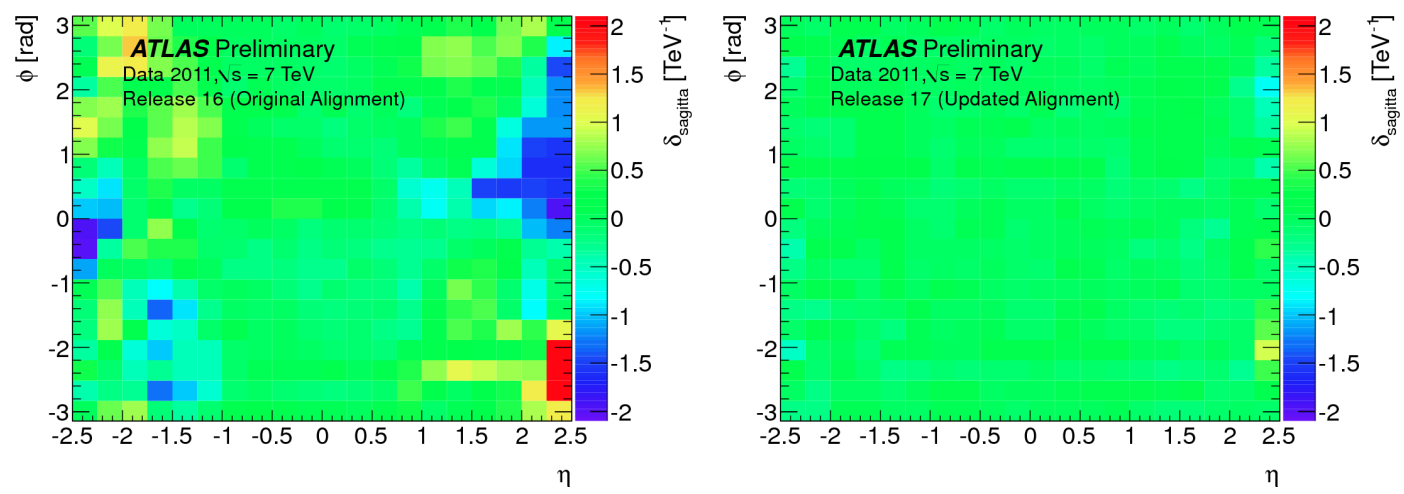

Figure 6: Momentum bias parameter for the charge-antisymmetric parametrization with old (left) and updated alignment (right) [5].

best performance possible in this challenging environment. Studies of systematic biases due to misalignment were also performed and showed improvements in momentum and mass measurements after corrections were applied.

\section{References}

[1] ATLAS Collaboration, The ATLAS Experiment at the CERN Large Hadron Collider, JINST 3 S08003 (2008).

[2] L. Evans and P. Bryant (editors), LHC Machine, JINST 3 S08001 (2008).

[3] ATLAS Collaboration, ATL-COM-PHYS-2012-474, http://atlas.web.cern.ch/Atlas/GROUPS/PHYSICS/IDTRACKING/PublicPlots/ATL-COM-PHYS2012-474/.

[4] ATLAS Collaboration, Performance of the ATLAS Inner Detector Track and Vertex Reconstruction in the High Pile-Up LHC Environment, ATLAS-CONF-2012-042, http://cds.cern.ch/record/1435196.

[5] ATLAS Collaboration., Study of alignment-related systematic effects on the ATLAS Inner Detector track reconstruction, ATLAS-CONF-2012-141, http://cds.cern.ch/record/1483518. 